\title{
A Method of Knowledge Based Measurement Points Distribution in Three-Dimensional Inspection Planning
}

\author{
Guijiang Duan ${ }^{1, a}$, Jiang'ao Zhao ${ }^{2, b}$ \\ ${ }^{1}$ School of Mechanical Engineering and Automation, Beihang University, Beijing, 100191, China \\ ${ }^{2}$ School of Mechanical Engineering and Automation, Beihang University, Beijing, 100191, China \\ aemail: gjduan@buaa.edu.cn, bemail:oceanzja@gmail.com
}

Keywords: Measurement point Planning; Inspection Plan; Knowledge; Three-Dimensional

\begin{abstract}
Aiming at promoting the efficiency of inspection planning and improving the integration between digital measurement equipment such as CMM (Coordinate Measurement Machine) and CAD system, a method of measurement-points distribution based on knowledge in threedimensional inspection planning was discussed in this paper. The algorithm of measurement-points arrangement directly based on three-dimensional CAD models was put forward. Furthermore, a measurement-points distribution tool was developed and integrated with the three-dimensional inspection planning system on Pro/E CAD platform. A case study was also given to validate the effectiveness of the method.
\end{abstract}

\section{Introduction}

Computer Aided Inspection Planning (CAIP) plays an important role in modern manufacturing systems, which aims as providing effective and efficient inspection strategy to control or evaluate the quality of parts or products. Traditional inspection planning was generally issued based on two-dimensional drawings. Recent years, with the rapid development of MBD (Model Based Definition), there is an obviously evolution in the fields of manufacturing system to change the traditional two-dimensional working styles to three-dimension environment. The requirement of three-dimensional based CAIP system becomes more urgent [1].

Measurement point planning is an important part of the three-dimensional CAIP system, which plays an important role in driving the digital inspecting equipment such as CMM [2] [3]. Currently, most of the measurement point plans were established on-line by the CMM operation system such as PC-DMIS or Rational-DMIS, which could not save the measurement points' information in the Three-dimensional CAD environment such as Pro/E or CATIA [4]. Thus it was impossible for inspection department to check and keep on drawing up the inspection plan. In other words, there is a gap between measurement point planning and three-dimensional CAD models.

To solve the problems above, a method of measurement point planning on three-dimensional inspection planning was put forward and studied in this paper. Additionally, a knowledge database was constructed to facilitate the measurement points planning process.

\section{Measurement Point Planning}

Measurement point was the only geometry elements that digital inspecting equipment can be identified. Information contained in the measurement point was shown on Fig. 1.

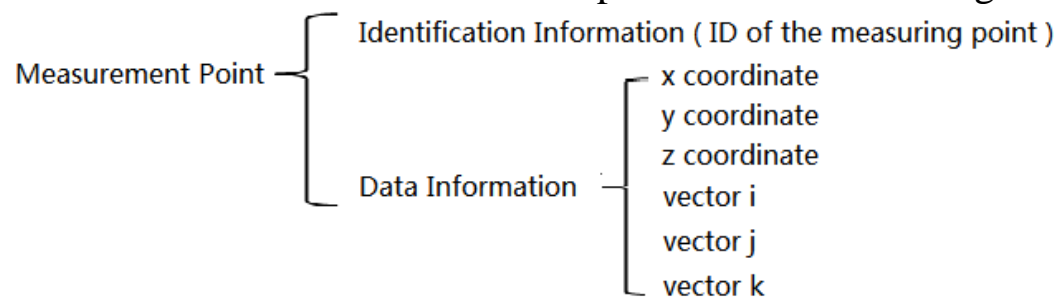

Fig.1. Information Contained in Measurement Point

From the geometric point of view, a surface is made up of a series of bounded two degree of 
freedom points' trajectories. It could be described as formula (1) [5]. Based on this formula, a distribution strategy on the basis of knowledge database is raised below.

$$
F(u, v)=(x(u, v), y(u, v), z(u, v))((u, v) \in D)
$$

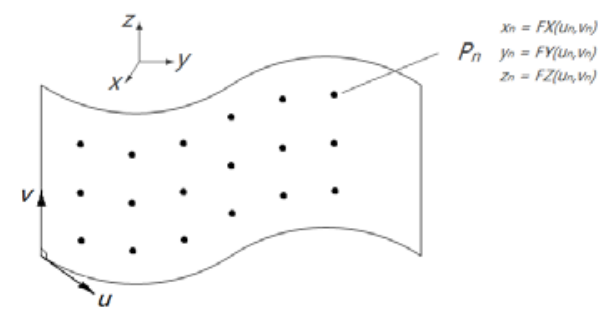

Fig.2. Measurement-points Distribution

The position and number of measurement points would not only affect the efficiency of the measuring device, but also affect the authenticity of the entire measurement results.[6] Therefore, distribution strategy is the core of the measurement point planning. It solved the problem that how to set measurement points on specific surface to ensure the inspecting accuracy to the most extent. Which means that under the same measurement environment and for the same feature, arranging the locations and number of the measurement points to ensure that it could truly reflect the geometry characteristics of the feature and could accurately evaluate the feature's tolerance information.

\section{Knowledge Based Measurement Points Planning}

To make measurement point planning much more simplified, a knowledge database is arranged, and in this knowledge database most feature types and rules are encapsulated. Feature types contained plan, holes, rib and exterior margin and the exterior margin could be expanded by the users. All the information of the rules was come from the standard of the manufactory industry.

Knowledge based distribution is one of the most intelligent distribution strategy. It is to matching the feature to a specific knowledge in the knowledge database, according to which the measurement points were distributed.

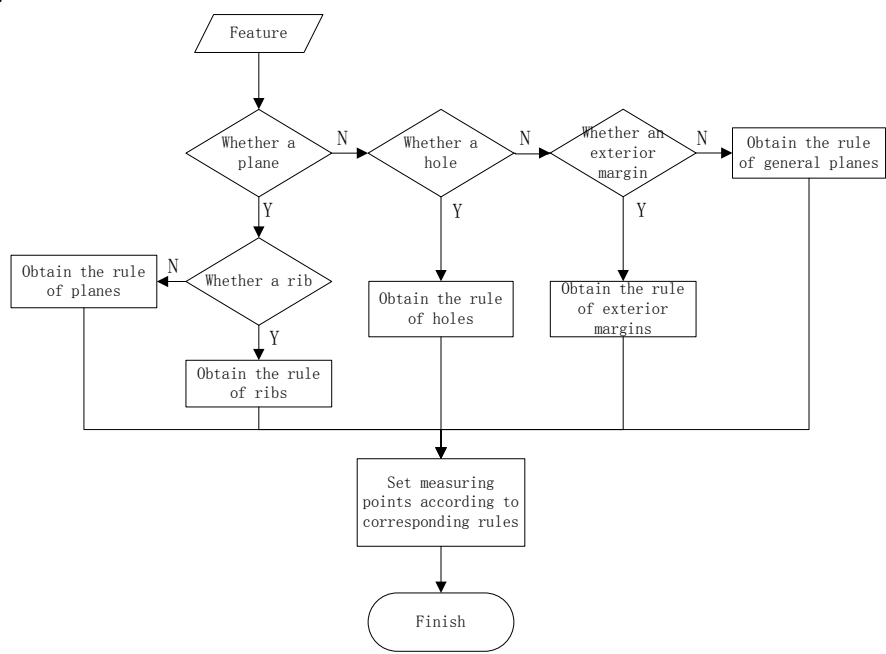

Fig.3. Flow Chart of Knowledge Based Distribution

The specific flow was shown in Fig.3. Initially, the feature was identified by the measurement point planning system. Secondly, the information such as the area and tolerance requirements was gained, which was the basic data of the knowledge based distribution. Then, by analyzing the data and matching the rules, a distribution strategy was generated. Finally, the measurement points were set and displayed on the CAD environment.

\section{Algorithm Analysis}

Two kinds of algorithms are put forward here. The first algorithm is reticular pointing and the second one is wavy pointing, shown as the following Fig.4 (a) and (b). 


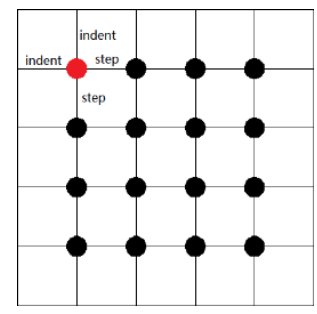

(a) Reticular Pointing

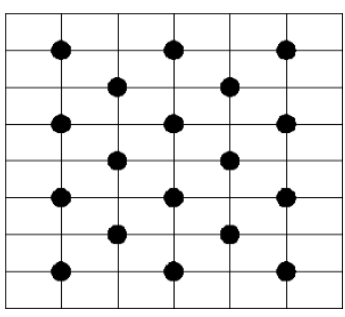

(b) Wavy Pointing

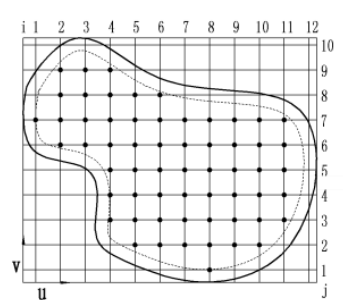

(c) Example of Reticular Pointing

Fig.4. Algorithm and Example

Reticular Pointing algorithm was the most basic algorithm, the distribution of the points were shown in Fig.4 (a). The distance of adjacent measuring points was called the step and the distance of the first point to the boundary was called the indent. Generally, a space curved surface can be described in the $\mathrm{u}, \mathrm{v}$ coordinate system which is obviously a two-dimensional coordinate system. An envelope surface was constructed on the basis of $u_{\min }, v_{\min }, u_{\max }$ and $v_{\max }$ of the space curved surface which was shown in Fig.4 (c). The measurement point could be described by formula (2)

$$
P(i, j)=\left(u_{i}+i \times u_{s}, v_{i}+j \times v_{s}\right)
$$

where $\mathrm{u}_{\mathrm{i}}, \mathrm{v}_{\mathrm{i}}$ were the indents on direction $\mathrm{u}$ and $\mathrm{v} ; \mathrm{u}_{\mathrm{s}}, \mathrm{v}_{\mathrm{s}}$ were the steps on direction $\mathrm{u}$ and $\mathrm{v}$.

Wavy Pointing is superior to Reticular Pointing in randomness. As for a cylindrical feature, under the condition of the same number of measurement points, there were more layers than reticular pointing. Thus the efficiency and accuracy is better than the former algorithm. The measurement point could be described by formula (3)

$$
P(i, j)=\left(u_{i}+\left(\frac{5}{4}+\frac{(-1)^{i}}{4}\right) i \times u_{s}, v_{i}+j \times v_{s}\right)
$$

where $\mathrm{u}_{\mathrm{i}}, \mathrm{v}_{\mathrm{i}}$ were the indents on direction $\mathrm{u}$ and $\mathrm{v} ; \mathrm{u}_{\mathrm{s}}, \mathrm{v}_{\mathrm{s}}$ were the steps on direction $\mathrm{u}$ and $\mathrm{v}$.

To ensure the distance of the adjacent two measurement points a constant value, the relationship of $u_{s}, v_{s}$ was described by formula (4).

$$
\left|v_{s}\right|=\frac{\sqrt{3}}{2}\left|u_{s}\right|
$$

\section{Case Study}

The part in Fig.5 (a) was designed by Pro/E. A measurement point plan was organized by the measurement point planning system. Fig.5 (b) was the knowledge based distribution. By matching the designated surface with the knowledge database, an optimizing distribution strategy was given and the measurement points would be distributed on the surface.

The organization of the inspection plan was shown in Fig.5 (c), where the measurement points are all saved in this plan. Fit.5 (d) showed the route planning system, after loading the measurement points, an optimizing route was calculated and displayed on the CAD environment. From the report display, all the information that measurement point contained could be checked, which was shown in Fig.5 (e). After organizing the inspection plan, a PC-DMIS program would be generated, by which could drive the digital inspecting equipment to finish the inspection automatically. 


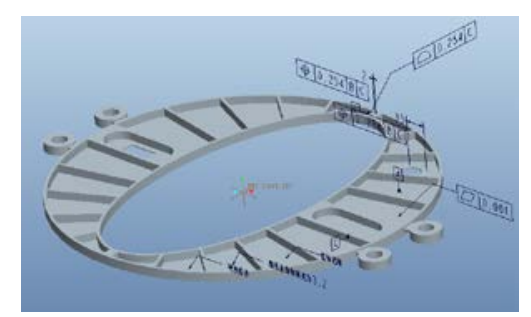

The Part Designed in Pro/E

(a)

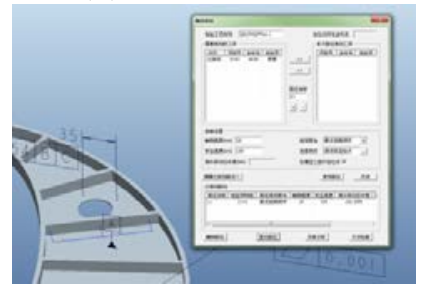

Route Plan Menu

(d)

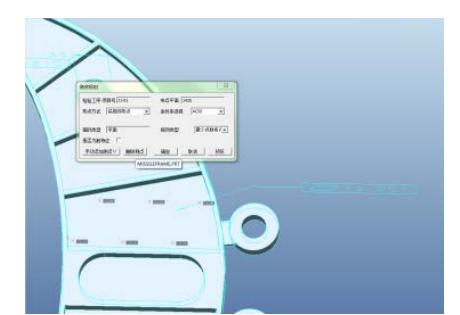

Knowledge Based Distribution

(b)

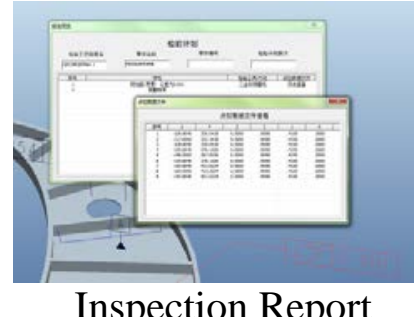

(e)

Fig.5. Examples

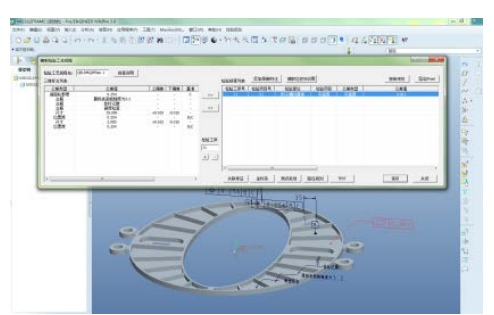

Inspection Plan Menu

(c)

\section{Conclusion}

Through the research on the process of measurement-points planning, algorithms about point plan were raised based on the actual engineering. By means of this algorithm, a measurement point planning system based on knowledge database was set up. Quality inspection personnel could easily organizing measurement point planning by this system. All of the information was attached to the three-dimensional CAD models, which made it possible for compilation and modification through CAD software.

\section{References}

[1] Jiang Zhang, Guijiang Duan. Research and System Development on Three-dimensional Inspection Planning for Typical Aircraft Structural Parts[J]. Aviation Precision Manufacturing Technology, 2002, 5: 30-39.

[2] Hwang C. Y., Tsai C. Y., Chang C. A. . Efficient inspection planning for coordinate measuring machines[J]. Int J Adv Manuf Technol, 2004, 23: 732-742.

[3] Tang X. Q., Davies B. J.. Integration of Inspection Planning System and CMM via DMIS[J]. The International Journal of Advanced Manufacturing Technology, 1995, 10(6): 422-426.

[4] Fan K. C., Leu M. C.. Intelligent planning of CAD-directed inspection for coordinate measuring machines [J]. Computer Integrated Manufacturing Systems, 1998, 11(1-2): 43-51.

[5] Cen Rong. Research on Detection Path Planning and Simulation Based on Three-Dimensional Model [D]. Beihang University, Master's Thesis, 2013.

[6] Zheng Weilian. Study on Inspection Data Analysis and Visualization Technology based on Three-Dimensional Model. 